FORUM EMPRESARIAL

Vol. 19. Núm. 2 • invierno 2014

\title{
El tamaño empresarial como factor que influye en el comportamiento innovador de las empresas mexicanas: un caso de estudio
}

\author{
José Luis Esparza Aguilar / jlesparza@uqroo.mx \\ Universidad de Quintana Roo, México \\ Teodoro Reyes Fong / teodoro_reyesf@ucol.mx \\ Universidad de Colima, México
}

Recibido: 21 de octubre de 2013

Aceptado: 28 de octubre de 2014

RESUMEN

El objetivo del trabajo es analizar el tamaño empresarial como factor que influye en el comportamiento innovador de las empresas. Se ha aplicado una encuesta a una muestra de 221 empresas en el sur del estado de Quintana Roo, México. Dentro de las variables de estudio se ha considerado a la innovación como la variable dependiente; y al tamaño empresarial, medido a través del empleo y las ventas, como la variable que influye en ese comportamiento. Para el análisis de los datos se han utilizado técnicas no paramétricas. Los resultados obtenidos muestran que el tamaño empresarial condiciona significativamente el comportamiento innovador de las empresas, y que las empresas de mayor tamaño son las que realizan mayor innovación.

Palabras clave: tamaño empresarial, pequeñas empresas, comportamiento innovador, Quintana Roo.

\section{ABSTRACT}

The purpose of this work is to analyze the enterprise size as a factor that influences the innovative behavior of enterprises. A survey of a sample of 221 businesses from the southern part of the state of Quintana Roo, México was used. Among the variables considered in the study, innovation of the business was used as the dependent variable. The enterprise size was used as the independent variable. For the data analysis, non parametric techniques were used. The results show that enterprise size significantly impacts the innovative behavior of businesses, and that larger businesses are more innovative.

Keywords: enterprise size, small businesses, innovative behavior, Quintana Roo. 


\section{INTRODUCCIÓN}

Ha sido creciente el interés de los gobiernos de diversos países por lograr ventajas competitivas en sus economías que les permitan alcanzar un crecimiento económico sostenible, equiparable o mayor que el de sus homólogos. Una de las formas a que se recurre con más frecuencia para lograr estas ventajas competitivas es el desarrollo de una mejor capacidad de innovar, o mejor dicho de generar nuevos productos, diseños, procesos, servicios, métodos u organizaciones, o de incrementar valor a los existentes (PNI, 2011).

Esta ventaja competitiva en México se logra a través de las micro, pequeñas y medianas empresas (Mipymes), debido a que son las principales detonantes de su desarrollo económico. Según datos del Instituto Nacional de Estadística y Geografía (INEGI, 2010), el $95.2 \%$ son microempresas, las pequeñas empresas son el $4.3 \%$ y las medianas empresas el $0.3 \%$. En Quintana Roo, las Mipymes representan aproximadamente el $99 \%$ de las unidades económicas.

La innovación es una variable que forma parte de los factores competitivos relevantes al que se enfrentan de manera cotidiana las pequeñas empresas, para poder desarrollarse (OCDE \& Eurostat, 2006). Diversos autores alrededor del mundo han estudiado esta temática y son parte de la revisión de la literatura. Es importante mencionar que en México, y por tanto en la región del sureste, son muy limitados los estudios empíricos que han investigado el tamaño y el comportamiento innovador de las empresas.

Aunado a lo anterior, una revisión de la literatura muestra diversos trabajos que abordan este tema, pero que llegan a resultados diversos (Aiken, Bacharach \& French, 1980; Hage, 1980; Damanpour, 1992). En este tenor es importante continuar generando conocimiento sobre diversos factores que les permitan a las empresas crecer y consolidarse. Con estos argumentos, se ha planteado la siguiente pregunta de investigación: ¿el tamaño es un factor que influye de manera significativa y positiva en el comportamiento innovador de las empresas mexicanas?

El objetivo principal del presente trabajo es analizar el tamaño empresarial como un factor que influye en el comportamiento 


\begin{abstract}
EL TAMAÑO EMPRESARIAL COMO FACTOR QUE INFLUYE EN EL COMPORTAMIENTO INNOVADOR...
\end{abstract}
innovador de las empresas, en donde reviste gran importancia realizar este tipo de investigación que ha sido muy escasa en un país con economía emergente, con la intención de contrastar estos resultados con los obtenidos por estudios previos en diversos contextos, y además por la gran relevancia que tienen estas empresas por su alta contribución al Producto Interno Bruto y a la generación de empleo en la región y el País. Asimismo, se pretende divulgar los resultados obtenidos a los diversos actores involucrados (académicos, empresarios y gobierno), para que comprendan la importancia de llevar a cabo actividades de innovación como un factor clave que influye en el desarrollo competitivo de estas empresas. Para llevar a cabo este trabajo, se ha realizado un estudio empírico de corte transversal con una muestra de 221 empresas en el sur del estado de Quintana Roo, México.

Basado en el objetivo del presente trabajo, el estudio se ha organizado de la siguiente manera: en primer lugar, se presenta la revisión teórica y los estudios empíricos previos; en segundo lugar, se describe la metodología de investigación y la muestra utilizada, así como la medición de variables; en tercer lugar, se proporciona el análisis de los resultados; y finalmente, se presentan las principales conclusiones del estudio, incluyendo limitaciones, implicaciones y futuros trabajos de investigación.

\title{
MARCO TEÓRICO: REVISIÓN DE ESTUDIOS EMPÍRICOS PREVIOS E HIPÓTESIS
}

La innovación definida en el Manual de Oslo se concibe como la introducción de un nuevo o significativamente mejorado producto (bien o servicio), de un proceso, de un nuevo método de comercialización o de un nuevo método organizativo, en las prácticas internas de la empresa, la organización del lugar de trabajo o las relaciones externas (OCDE \& Eurostat, 2006).

Actualmente, diversos agentes económicos como empresas, universidades y organismos públicos dedicados a la investigación de un país o región son los que realizan las actividades de innovación y desarrollo tecnológico; sin embargo, es bastante compleja la implementación de un proceso de innovación, especialmente en las 
pequeñas empresas ya que tienen diversas limitaciones en cuanto a recursos financieros, humanos y tecnológicos (Hadjimanolis, 1999), lo que origina una menor inversión en este concepto, principalmente en el desarrollo de nuevos productos o servicios (Larsen \& Lewis, 2007).

Sin duda alguna y a raíz de la apertura de los mercados internacionales, las empresas deben buscar la manera de sobrevivir, crecer y ser rentables, y para lograrlo deben realizar actividades de innovación (Freel, 2005). La innovación es considerada en las pequeñas y medianas empresas (pymes) como un elemento fundamental para su desarrollo y crecimiento. Llevar a cabo innovación está relacionado directamente con la ventaja competitiva, entendida ésta como el nivel de desarrollo que se tiene en una determinada área o región (García Docampo, 2007).

Por necesidad, las pymes desarrollan actividades más especializadas. Es de máxima importancia para ellas interactuar de manera eficiente con las otras empresas y los establecimientos públicos de investigación de I+D, en el intercambio de conocimientos y, eventualmente, en las actividades de comercialización (OCDE \& Eurostat, 2006).

Es importante mencionar que la competitividad de la economía mexicana y el bienestar de sus trabajadores dependen en buena parte del éxito de las pequeñas empresas; sin embargo, estas empresas encuentran diversos obstáculos para desarrollar o realizar innovaciones, principalmente, por las limitaciones de sus recursos y del mercado en el que se desarrollan, al no disponer en la mayoría de los casos de infraestructura y equipamiento para realizar investigación, así como su difícil acceso a los recursos financieros (Ruber \& Fuentes, 2003).

La innovación realizada en las pequeñas empresas trae consigo muchos beneficios para el desarrollo socioeconómico de una región. Durante este proceso, se profesionaliza la gestión a través del capital humano, lo que trae como consecuencia importantes mejoras en la productividad de las empresas. Además, dada la movilidad que existe en el mercado laboral, es razonable esperar que este factor humano sea compartido con otras empresas y con 
EL TAMAÑO EMPRESARIAL COMO FACTOR QUE INFLUYE EN EL COMPORTAMIENTO INNOVADOR...

la economía de manera integrada (Canales, Glover, González \& Kessler, 2009; PNI, 2011).

La innovación se percibe como un elemento del proceso de acumulación de capital que empuja a las pequeñas empresas a mejorar su productividad, al menos por arriba de los estándares de sus competidores, para permanecer o posicionarse en los mercados competitivos. Este empuje hacia la innovación y el desarrollo tecnológico es el factor clave del desenvolvimiento del capitalismo $y$, por ende, explica en gran medida las diferencias entre los diversos entes económicos (Morales, 2011).

En cuanto al tamaño empresarial y la innovación, la revisión de la literatura previa se remonta a las hipótesis teóricas establecidas por Schumpeter (1944) y Galbraith (1952), las cuales dieron una mayor importancia a las empresas de mayor tamaño; en sentido contrario, las empresas de menor tamaño desarrollan menos actividad innovadora, ya que éstas cuentan con una limitada disponibilidad de recursos para competir en los diferentes mercados.

Diversos estudios obtienen diferentes resultados relacionado con el tamaño de la empresa y la innovación. Por un lado, las ventajas competitivas de las pequeñas empresas se orientan en la flexibilidad y en su alta capacidad de cambio y adaptación, así como un diseño organizativo más práctico; sin embargo, la empresa de mayor tamaño tiene mayores recursos, más capacidad de crecimiento y un mayor control sobre variables del entorno en mayor medida que las pequeñas empresas (Dean, Brown \& Bamford, 1998; Camisón, 2000).

El Programa Nacional de Innovación del Gobierno Mexicano (2011) establece que una de las principales características de las empresas que ocupan hasta a 10 personas es que en general no son innovadoras, debido a que utilizan infraestructura que en ocasiones es obsoleta y de baja calidad, además de que desarrollan una comercialización poco adecuada. Si bien el número de empresas de este tipo es elevado, su contribución al valor total de la producción es baja, particularmente en los sectores de manufacturas y servicios.

En México, se hizo un estudio con pymes de los estados de Aguascalientes, Coahuila, Colima, Durango, Hidalgo, Nayarit, Quintana Roo, San Luis Potosí y Tabasco, relacionado con las 
hipótesis de que la empresa hacia la innovación está relacionada con el tamaño. Los resultados fueron que la búsqueda de la innovación va dependiendo del tamaño, posibilitando una mayor estructura el poder optar por un perfil más innovador (López, Somohano \& Martínez, 2011).

Basado en la revisión de la literatura previa, en donde se establece la existencia de un efecto positivo que señala al tamaño de la empresa como el mejor predictor del comportamiento innovador (Moch \& Morse, 1977; Sullivan \& Kang, 1999; Aragón \& Cordón, 2000; Estrella \& Góngora, 2009; Vila, Ferro \& Guisado, 2010; López, Somohano \& Martínez, 2011), se plantean las siguientes hipótesis a contrastar:

$\mathrm{H}_{1}$. El tamaño empresarial es un factor que influye de manera significativa en el comportamiento innovador de las empresas.

$\mathrm{H}_{2}$. Las empresas cuanto mayor sea su tamaño empresarial, mayor será su comportamiento innovador.

$\oplus$

\section{Metodología de LA investigación}

\section{Selección de la muestra}

Basado en cifras de los Censos Económicos 2009 del Instituto Nacional de Estadística y Geografía (INEGI, 2010), se ha extraído la población de las Mipymes del estado de Quintana Roo. De acuerdo a esta información, existen 25,675 unidades económicas que se resumen en la Tabla 1.

Tabla 1

Distribución de las Mipymes en Quintana Roo

\begin{tabular}{|c|c|c|c|c|c|}
\hline \multirow[t]{2}{*}{ Empresa } & \multicolumn{4}{|c|}{ Sector de actividad } & \multirow[t]{2}{*}{ Total } \\
\hline & $\underline{\text { Manufactura }}$ & $\underline{\text { Comercio }}$ & $\underline{\text { Servicios }}$ & Construcción & \\
\hline Micro & 2,630 & 17,562 & 3,537 & 119 & 23,848 \\
\hline Pequeña & 150 & 796 & 422 & 152 & 1,520 \\
\hline Mediana & 19 & 144 & 92 & 52 & 307 \\
\hline Total & 2,799 & 18,502 & 4,051 & 323 & 25,675 \\
\hline
\end{tabular}

Fuente: Elaboración propia basada en INEGI (2010).

36 ISSN 1541-8561 FORUM EMPRESARIAL VOL. 19. NÚM. 2 INVIERNO 2014 
EL TAMAÑO EMPRESARIAL COMO FACTOR QUE INFLUYE EN EL COMPORTAMIENTO INNOVADOR...
\end{abstract}

Para desarrollar esta investigación de tipo descriptiva y exploratoria, se realizó un análisis de corte transversal con una muestra de 221 empresas. El tamaño se ha considerado de acuerdo a los criterios de estratificación de empresas establecida en la Ley para el Desarrollo y Competitividad de la Micro, Pequeña y Mediana Empresa, artículo $3^{\circ}$ Fracción III, del Gobierno Federal de México (DOF, 2009).

Las características de las empresas objeto de estudio son: principalmente micro (hasta 10 trabajadores) y pequeñas (entre $11 \mathrm{y}$ 50 trabajadores); con una antigüedad promedio de 10 años en el mercado; la edad promedio del gerente-propietario es de 42 años, con una experiencia media en este tipo de negocio de 11 años; y la mayoría de las empresas son de propiedad familiar. Lo anterior se decidió, en virtud de que este tipo de empresas son las que mayormente predominan en la región y, principalmente, son las que requieren mayor atención académica por su constante problemática a la que se enfrentan día con día. La micro empresa se dividió en dos niveles de agrupación, para poder observar y diferenciar aquéllas que por su número de trabajadores, tienen un mayor o menor comportamiento hacia las actividades de innovación.

El diseño general de la muestra se basó en los principios del muestreo aleatorio simple en poblaciones finitas. Partiendo del total de la población objetivo y adoptando el porcentaje de error de muestreo estándar aceptado en este tipo de encuestas se asume un nivel de significación del 95\%; y considerando el caso más sencillo de muestreo aleatorio simple para estimaciones de proporciones de población, el error de muestreo $\varepsilon$ estará determinado por la siguiente expresión:

$$
\varepsilon=\kappa \sqrt{\frac{P Q}{n} * \frac{N-n}{N-1}}
$$

Donde $\mathrm{P}$ es la proporción de la población que se pretende estimar, siendo $\mathrm{Q}=100-\mathrm{P}, \mathrm{N}$ es el tamaño de la población total, $\mathrm{n}$ es la muestra utilizada, у $\mathrm{\kappa}$ es el valor de una distribución normal correspondiente a un nivel de confianza del $95 \%$, por lo que $\kappa=1.96$. Despejando de la expresión anterior y fijando a priori un error de muestreo 
máximo aceptable del $5.2 \%$, la muestra necesaria para inferir el comportamiento del total de la población de Mipymes, se determinó partiendo de la siguiente expresión:

$$
\mathrm{n}=\frac{\mathrm{K}^{2} \mathrm{PQN}}{\varepsilon^{2}(\mathrm{~N}-1)+\mathrm{K}^{2} \mathrm{PQ}}
$$

Para determinar las proporciones se parte del caso más aceptado en este tipo de estudios empíricos, que es suponer que la población se distribuye exactamente al $50 \%$, es decir que $\mathrm{P}=50$ y $\mathrm{Q}=50$, por lo que el tamaño de la muestra es de 350 .

Aun cuando el tamaño de la muestra estimada fue de 350 observaciones, al final del proceso el total de encuestas válidas quedó en 221; por lo tanto, con una muestra de 221 observaciones el error de muestreo permitido se incrementó al $6.4 \%$, límite válido en estudios de las Ciencias Sociales. La distribución de la muestra final utilizada se puede observar en la Tabla 2.

Tabla 2

Distribución de la muestra

\begin{tabular}{|c|c|c|c|c|c|}
\hline \multirow[t]{2}{*}{$\begin{array}{l}\text { Empresa por número } \\
\text { de trabajadores }\end{array}$} & \multicolumn{4}{|c|}{ Sector de actividad } & \multirow[t]{2}{*}{ Total } \\
\hline & $\underline{\text { Manufactura }}$ & $\underline{\text { Comercio }}$ & $\underline{\text { Servicios }}$ & $\underline{\text { Otros }}$ & \\
\hline De 1 a 5 & 7 & 145 & 42 & 7 & 201 \\
\hline De 6 a 10 & & 8 & 5 & 1 & 14 \\
\hline De 11 a 50 & 1 & 4 & 1 & & 6 \\
\hline Total & 8 & 157 & 48 & 8 & 221 \\
\hline
\end{tabular}

Fuente: Elaboración propia.

La técnica utilizada para la obtención de datos y determinar la existencia de relaciones de causa y efecto entre los fenómenos del estudio ha sido la encuesta (Creswell, 2005; Hernández, Fernández \& Baptista, 2006). El instrumento de medición utilizado fue un cuestionario dirigido directamente a los directores, gerentes y propietarios de las empresas, considerando las características estructurales de las Mipymes y la constante carencia de bases de 


\begin{abstract}
EL TAMAÑO EMPRESARIAL COMO FACTOR QUE INFLUYE EN EL COMPORTAMIENTO INNOVADOR...
\end{abstract}
datos económicas en México. El cuestionario ayuda a conocer las ideas y opiniones de los encuestados acerca de los aspectos relativos al estudio, de manera directa y simple (Sabino, 1984), proporciona flexibilidad, relativa facilidad y rapidez al momento de aplicarlo y procesarlo, y es particularmente útil para estudios descriptivos no experimentales (Mathers, Fox \& Hunn, 1998).

El diseño del cuestionario se efectuó a partir de la revisión de estudios empíricos previos tratando de recoger las variables definidas para alcanzar los objetivos establecidos. Una vez integrado el cuestionario, se efectuó en primer lugar, una prueba piloto del mismo, compuesto por diversos académicos conocedores del tema. Posteriormente, y con la finalidad de ajustar el cuestionario a la realidad de la empresa, se efectuó una segunda prueba piloto a 10 Mipymes. Después de las pruebas respectivas, se realizaron las modificaciones pertinentes al instrumento con el fin de adicionar, corregir o aclarar las diversas preguntas planteadas. La validez y fiabilidad de las escalas del cuestionario se verificaron, a través de la validez de contenido, constructo y criterio, así como la consistencia interna de los ítems por medio del coeficiente alfa de Cronbach.

Para llevar a cabo la encuesta y con el propósito principal de conseguir la cooperación y disposición del director, gerente y propietario a participar en la investigación, se elaboró una carta de presentación de la Universidad de Quintana Roo, con la finalidad de formalizar y dar a conocer de manera general el alcance del trabajo a realizar. Los datos de las empresas se obtuvieron del directorio electrónico de empresas afiliadas al Sistema Empresarial Mexicano (SIEM). Las solicitudes para contactar a los entrevistados se realizaron a través de llamadas telefónicas y por correo electrónico, logrando un total de 350 citas. Al final se obtuvieron 221 encuestas válidas, lo que supone una tasa de respuesta del $64.11 \%$. El trabajo de campo se realizó en el sur del estado de Quintana Roo, en el municipio de Othón P. Blanco, entre los meses de enero a marzo de 2011.

Finalmente, para la recogida de la información participaron profesores integrantes del Departamento de Ciencias Económico Administrativas, adscrito a la División de Ciencias Sociales y Económico Administrativas (DCSEA) de la Universidad de Quintana 
Roo, así como estudiantes de las carreras de Sistemas Comerciales, y de Economía y Finanzas, quienes fueron capacitados en tiempo y forma para llevar a cabo la encuesta establecida, y también para garantizar que la información obtenida fuera utilizada de manera anónima, con estricta confidencialidad y únicamente para los fines de la investigación. Asimismo, el tratamiento estadístico de los datos se realizó con el programa informático SPSS.

\section{Medición de VARIABLES}

Variable dependiente: Comportamiento innovador. La innovación en las pequeñas empresas es un elemento muy importante para su desarrollo (García Docampo, 2007; Lin \& Huang, 2008); por tal motivo, se ha medido esta variable a través de una escala de Likert de 5 puntos (1 poca importancia y 5 mucha importancia) en donde se ha preguntado al director-gerente-propietario las áreas de su empresa donde se han efectuado innovaciones en productos y servicios, comercialización, procesos, gestión, TIC, calidad, y compras y ventas de su empresa en los dos últimos años, así como la importancia que le da a las mismas. La validez y fiabilidad de estas medidas se verificaron a través del coeficiente alfa de Cronbach (0.896) y se comprobó, mediante un análisis factorial, que los indicadores anteriores se han resumido en un solo factor, capaz de reflejar adecuadamente la importancia considerada $(66.748 \%$ varianza total explicada). Finalmente y para el objetivo de la investigación, se ha construido una variable binaria que toma valor 1 cuando la empresa es poco innovadora, y valor 2 cuando es muy innovadora.

Variable explicativa: Tamaño empresarial. Debido a la falta de indicadores en nuestra región y país acerca del tamaño empresarial, se han utilizado dos criterios para su definición: el número de trabajadores y el monto de las ventas. La variable empleo ha sido medida a través del número total de trabajadores de la empresa durante el periodo estudiado (Damanpour, 1992). Asimismo, se ha medido el monto de las ventas de la empresa, a través de una escala ordinal que va desde un monto de 1 millón hasta 100 millones de pesos (mexicanos). La variable para medir el tamaño empresarial 
EL TAMAÑO EMPRESARIAL COMO FACTOR QUE INFLUYE EN EL COMPORTAMIENTO INNOVADOR...

con estos dos criterios ha sido utilizada por Vila, Ferro y Guisado (2010).
\end{abstract}

\title{
AnÁlisis de los Resultados
}

Para el análisis de los datos y la obtención de resultados, el presente trabajo se centró en los procedimientos de la estadística no paramétrica, debido a que la eficiencia de estos procedimientos es bastante alta para muestras pequeñas (Hair, Anderson, Tatham \& Black, 2005), como el estudio en mención. Estas pruebas generalmente se aplican con mayor frecuencia a los datos nominales y ordinales, en virtud de que no existen supuestos sobre la distribución de los parámetros de la población; por lo anterior, a continuación se presenta un análisis no paramétrico aplicable a esta investigación.

Para comenzar y en virtud de que la variable comportamiento innovador es binaria o dummy, se puede obtener una aproximación de dicho comportamiento antes de proceder a realizar los contrastes finales de las hipótesis establecidas, a partir de los porcentajes que alcanzan cada una de sus dos alternativas. Así, en la Tabla 3 se ha obtenido que de un total de 221 empresas de las que consta la muestra, el $75.2 \%$ (166) son poco innovadoras mientras que el $24.8 \%$ (55) son empresas muy innovadoras.

Tabla 3

\begin{tabular}{lccc} 
Comportamiento innovador y tamaño de la empresa \\
\hline $\begin{array}{l}\text { Tamaño por número } \\
\text { de trabajadores }\end{array}$ & $\begin{array}{c}\text { Empresas poco } \\
\text { innovadoras }\end{array}$ & $\begin{array}{c}\text { Empresas muy } \\
\text { innovadoras }\end{array}$ & Total \\
\hline De 1 a 5 & 159 & 42 & 201 \\
De 6 a 10 & 5 & 9 & 14 \\
De 11 a 50 & 2 & 4 & 6 \\
\hline
\end{tabular}

Fuente: Elaboración propia.

Por otra parte, los valores obtenidos del contraste Chi-cuadrado para tamaño empresarial, medido a través de las variables empleo y ventas se reflejan en la Tabla 4. 
Tabla 4

Tamaño empresarial y comportamiento innovador

\begin{tabular}{lccc}
\hline Tamaño empresarial & $\chi 2$ de Pearson & gl & Significación asintótica bilateral \\
\hline Empleo (trabajadores) & 655.538 & 16 & .000 \\
Ventas & 217.448 & 2 & .000 \\
\hline
\end{tabular}

Nota: Test Chi-cuadrado de Pearson.

Fuente: Elaboración propia.

El contraste Chi-cuadrado de Pearson demuestra que, a un nivel de confianza pleno, existe una relación significativa entre las variables que representan el tamaño empresarial y el comportamiento innovador. En este sentido, aunque ese contraste permite obtener una primera indagación sobre la asociación de las variables mencionadas, el resultado no es nada definitivo, ya que su nivel de eficacia disminuye cuando se aplica a variables ordinales, y su fiabilidad sobre el grado de significación de la relación resulta poco eficiente en grandes muestras.

Estas dos características están consideradas en el presente estudio, por lo que es necesario aplicar otro tipo de contraste complementario. Debido a que la variable comportamiento innovador es binaria y la variable tamaño empresarial, ordinal, el contraste adecuado a aplicar es el de diferencias de medias, aunque previamente es necesario comprobar que la variable tamaño sigue una distribución normal. Para esto se aplica la prueba de KolmogorovSmirnov (Tabla 5).

Tabla 5

Prueba de Kolmogorov-Smirnov

\begin{tabular}{lcc}
\hline Tamaño empresarial & Z de Kolmogorov-Smirnov & Significación asintótica bilateral \\
\hline Empleo (trabajadores) & 2.308 & .000 \\
Ventas & 1.599 & .012 \\
\hline
\end{tabular}

Fuente: Elaboración propia. 
EL TAMAÑO EMPRESARIAL COMO FACTOR QUE INFLUYE EN EL COMPORTAMIENTO INNOVADOR...

Con este resultado obtenido, la prueba de Kolmogorov-Smirnov permite, a un nivel de confianza total, rechazar la hipótesis nula de que la variable empleo y ventas sigue una distribución normal; por consiguiente, resulta inadecuado aplicar la prueba de diferencias de medias, por lo que es necesario aplicar otro tipo de prueba que no requiera esta clase de exigencias. Para esto, y considerando que la prueba no paramétrica más utilizada para contrastar asociaciones entre variables ordinales y que no tiene exigencias de normalidad sobre sus correspondientes funciones de distribución es el U de Mann-Whitney, se ha procedido a aplicar dicho contraste (Tabla 6).

Tabla 6

Prueba de Mann-Whitney

\begin{tabular}{lcc}
\hline Tamaño empresarial & U de Mann-Whitney & Significación asintótica bilateral \\
\hline Empleo (trabajadores) & $6,446.000$ & .000 \\
Ventas & $5,912.000$ & .000
\end{tabular}

Nota: Variable de agrupación "Empresas poco innovadoras y muy innovadoras". Fuente: Elaboración propia.

La prueba de Mann-Whitney corrobora la asociación entre variables obtenida inicialmente por el contraste Chi-cuadrado de Pearson; por lo tanto, con un nivel de confianza pleno, se puede aceptar la hipótesis relacionada en cuanto a que el tamaño empresarial es un factor que influye de manera significativa en el comportamiento innovador de las empresas $\left(\mathrm{H}_{1}\right)$.

Por otra parte, el contraste de Mann-Whitney no proporciona información relevante sobre el sentido de la relación entre variables, es decir, si las empresas de menor o mayor tamaño empresarial son las que tienen un menor o mayor comportamiento innovador; por consiguiente, para conocer el sentido de esa relación se aplican dos contrastes, el coeficiente de Kendall y el de Spearman, los cuales son utilizados frecuentemente con variables ordinales (Tabla 7 ). 
Tabla 7

Indices de Kendall y Spearman

\begin{tabular}{lcccc}
\hline Tamaño empresarial & $\begin{array}{c}\text { Tau-c de } \\
\text { Kendall }\end{array}$ & $\begin{array}{c}\text { Significación } \\
\text { aproximada }\end{array}$ & $\begin{array}{c}\text { Correlación de } \\
\text { Spearman }\end{array}$ & $\begin{array}{c}\text { Significación } \\
\text { aproximada }\end{array}$ \\
\hline Empleo (trabajadores) & .288 & .000 & .320 & .000 \\
Ventas & .310 & .000 & .318 & .000 \\
\hline
\end{tabular}

Fuente: Elaboración propia.

Es importante mencionar que los índices de Kendall y Spearman arrojaron datos positivos y estadísticamente significativos a un nivel de confianza total, lo que permite aceptar que las empresas de mayor tamaño empresarial, medido a través del empleo y las ventas, son las que tienen un mayor comportamiento innovador. Con estos resultados, la segunda hipótesis puede ser comprobada $\left(\mathrm{H}_{2}\right)$. Lo anterior se da en concordancia con los resultados obtenidos por diversos autores, en donde establecen una relación positiva entre el tamaño y el comportamiento innovador de las empresas (Damanpour, 1992; Aragón \& Cordón, 2000; Estrella \& Góngora, 2009; Vila, Ferro \& Guisado, 2010; López, Somohano \& Martínez, 2011).

En general, los resultados obtenidos corroboran lo hallado por otros estudios; sin embargo, es importante mencionar que estos resultados no pueden generalizarse a cada uno de los sectores económicos en que operan estas empresas, debido a que la zona sureste donde se ha llevado a cabo el estudio, es una zona limítrofe con Belice, América Central, en donde se tiene establecida una zona libre comercial, dedicada en su mayoría al comercio y al turismo natural y de bajo impacto, y en donde prevalecen los pequeños negocios, restaurantes y hoteles que le dan atención a la población local y a los visitantes. Por tal razón, se pudo observar que las empresas de 1 a 5 trabajadores son las que menos innovan con un $71.9 \%$ y representan al sector comercio con el $65.6 \%$, siguiéndole el sector servicios con un $19.0 \%$ del total de la muestra. Por consiguiente, aunque los resultados de este trabajo muestran una relación positiva entre el tamaño empresarial y el comportamiento innovador, se detectaron algunas observaciones con otras variables que bien 
EL TAMAÑO EMPRESARIAL COMO FACTOR QUE INFLUYE EN EL COMPORTAMIENTO INNOVADOR...

pueden influir en este comportamiento y que son tema para futuros trabajos de investigación, como el considerar la zona geográfica (norte) en donde se encuentran los sitios turísticos más importantes del estado de Quintana Roo, México (Cancún, la Riviera Maya y Tulum), y en donde se ubica la mayoría de las empresas de esta región, las cuales se desarrollan bajo un contexto de turismo internacional y de comercio de mayor impacto económico.
\end{abstract}

\title{
Conclusiones
}

En virtud de que las pequeñas empresas son muy importantes para el desarrollo económico de una región, la innovación es un factor crucial debido a que genera diversos beneficios para estas empresas, pues trae como resultado mayor rentabilidad derivado de la posibilidad de diseñar y producir nuevos o mejores bienes y servicios o de utilizar técnicas productivas más eficientes que la de su competencia. Además, cuentan con el conocimiento necesario para dar respuesta rápida y eficaz a las oportunidades de la apertura económica, así como responder eficientemente a las amenazas competitivas de sus rivales y del entorno; todo esto, traducido en la posibilidad de crecer de manera sostenida (PNI, 2011).

Por lo anterior, en este trabajo se han contrastado hipótesis relacionadas con que el tamaño empresarial condiciona de manera significativa el comportamiento innovador de las micro y pequeñas empresas en el sur del estado de Quintana Roo. Los resultados obtenidos son consistentes con otros estudios y muestran que el tamaño, medido a través del número de trabajadores y el monto de ventas, tiene un efecto positivo en el comportamiento innovador, de manera que las empresas de mayor tamaño tienden a ser más innovadoras y, por consiguiente, las de menor tamaño tienden a ser las que realizan menor innovación. Esto es un claro indicador de que las empresas de menor tamaño necesitan recursos, para poder invertir en actividades de innovación y así poder mantenerse de manera competitiva en sus respectivos mercados.

Dentro de las limitaciones que tiene esta investigación, se puede mencionar el estudio realizado exclusivamente a micro 
y pequeñas empresas, la representatividad geográfica regional, la continua falta de bases de datos económicas de empresas y la estratificación de empresas exclusivamente a base del número de trabajadores; por lo tanto, es importante realizar futuras investigaciones relacionadas con otras variables, tanto internas (nivel educativo del gerente y propietario, estrategia empresarial, antigüedad, cultura empresarial, uso de las TIC, investigación y desarrollo, acceso a financiamiento) como externas (desarrollo económico, competencia, diversidad geográfica, políticas públicas), que también influyen en el comportamiento innovador de estas empresas y que estudios previos así lo demuestran, principalmente estudios empíricos realizados en países con economías emergentes.

Finalmente, la información que proporciona este trabajo contribuye: al estado del arte, enriqueciendo la literatura existente relacionada con la investigación sobre el comportamiento innovador de las empresas en un país emergente y en un contexto donde prevalece la actividad turística y el comercio; a las instituciones gubernamentales, para que ayuden a potencializar las fortalezas y minimizar las debilidades de estas empresas, a través de políticas públicas orientadas a que las empresas realicen mayores actividades de innovación; a los gerentes y propietarios, a fin de que comprendan la importancia de implementar actividades innovadoras en sus procesos o en sus productos y servicios dentro de la empresa como un factor que les permitirá desarrollarse competitivamente; también, a las universidades, a efecto de que los resultados hallados se divulguen en los diferentes programas educativos relacionados con empresas o negocios; y, de igual forma, a los profesores investigadores de las universidades, para que continúen generando y aplicando conocimiento en estos temas tan importantes para la comunidad académica y empresarial. 
EL TAMAÑO EMPRESARIAL COMO FACTOR QUE INFLUYE EN EL COMPORTAMIENTO INNOVADOR...

\section{Referencias}

Aiken, M., Bacharach, S. B., \& French, J. L. (1980). Organizational structure, work process, and proposal making in administrative bureaucracies. Academy of Management Journal, 23(4), 631-652.

Aragón, J. A., \& Cordón, E. (2000). La influencia del tamaño, las dimensiones estratégicas y el entorno en la implantación de innovaciones en la organización: Internet en las pequeñas y medianas empresas. Investigaciones Europeas de Dirección y Economía de la Empresa, 6(2), 91-110.

Canales, D., Glover, A., González, M., \& Kessler, T. (2009). Apoyando la innovación en las pymes: El estado del arte. [México, DF:] Fundación IDEA.

Camisón, C. (2000). Reflexiones sobre la investigación científica de la pyme. Investigaciones Europeas de Dirección y Economía de la Empresa, 6(2), 13-29.

Creswell, J. (2005). Educational research: Planning, conducting, and evaluating quantitative and qualitative research (2nd ed.). Upper Saddle River, NJ: Person Education.

Damanpour, F. (1992). Organizational size and innovation. Organization Studies, 13(3), 375-402.

Dean, T. J., Brown, R. L., \& Bamford, C. E. (1998). Differences in large and small firm responses to environmental context: Strategic implications from a comparative analysis of business formations. Strategic Management Journal, 19(8), 709-728.

Diario Oficial de la Federación (DOF). (2009). Acuerdo por el que se establece la estratificación de las micro, pequeñas y medianas empresas. México, DF: Diario Oficial de la Federación.

Estrella, E., \& Góngora, G. (2009). La pyme innovadora mexicana. Revista Gestión Joven, 14-19.

Freel, M. (2005). Perceived environment uncertainty and innovation in small firms. Small Business Economics, 25, 49-64.

Galbraith, J. K. (1952). American capitalism. Boston, MA: HoughtonMiffing Co.

García Docampo, M. (2007). Perspectivas teóricas en desarrollo local. La Coruña: Netbiblo, S.L.

Hadjimanolis, A. (1999). Barriers to innovation for SMEs in a small less developed country (Cyprus). Technovation, 19, 561-570. 
Hage, J. (1980). Theories of organizations. Nueva York, NY: Wiley.

Hair, J. F., Anderson, R. E., Tatham, R. L., \& Black, W. C. (2005). Análisis multivariante (5a ed.). Madrid: Pearson Educación.

Hernández, R., Fernández, C., \& Baptista, P. (2006). Metodología de la investigación (4a ed.). México, DF: McGraw-Hill.

Instituto Nacional de Estadística y Geografía (INEGI). (2010). Censos económicos 2009. México, DF: Instituto Nacional de Estadística y Geografía.

Larsen, P., \& Lewis, A. (2007). How award-winning SMEs manage the barriers to innovation. Creativity and Innovation Management, 16(2), 142-151.

Lin, P., \& Huang, D. (2008). Technological regimes and firm survival: Evidence across sectors and over time. Small Business Economics, 30, 175-186.

López Fernández, J. M., Somohano Rodríguez, F. M., \& Martínez García, F. J. (2011). La innovación y la ventaja competitiva en las pymes mexicanas. Recuperado de http://personales.ya.com/ aeca/pub/on_line/comunicaciones_aal2011/cd/55k.pdf

Mathers, N., Fox, N., \& Hunn, A. (1998). Surveys and questionnaires. Notthingam Trent Focus Group, 1-50.

Moch, M. K., \& Morse, E. V. (1977). Size, centralization and organizational adoption of innovations. American Sociological Review, 42, 716-725.

Morales Nájar, I. (2011). Las pymes en México, entre la creación fallida y la destrucción creadora. Economía Informa, 366, 39-48.

Organización para la Cooperación y el Desarrollo Económicos (OCDE) y Eurostat (2006). Manual de Oslo. Guía para la recogida e interpretación de datos sobre innovación (3a ed.). España: Organización para la Cooperación y el Desarrollo Económicos y Eurostat.

Ortiz, F. (2006). Gestión de innovación tecnológica de las pymes manufactureras. En I Congreso Iberoamericano de Ciencia, Tecnología, Sociedad e Innovación CTS+ I, 1-17.

Programa Nacional de Innovación (PNI) (2011). Comité Intersectorial para la Innovación (SE). Recuperado de http://www. economia.gob.mx/files/comunidad_negocios/innovacion/ Programa_Nacional_de_Innovacion.pdf 


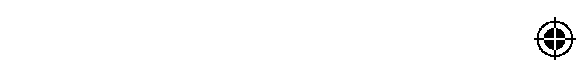

EL TAMAÑO EMPRESARIAL COMO FACTOR QUE INFLUYE EN EL COMPORTAMIENTO INNOVADOR...

Ruber, J. J., \& Fuentes, A. M. (2003). La economía regional en el marco de la nueva economía. Valencia: Asociación Valenciana de Ciencia Regional.

Sabino, C. (1984). Introducción a la metodología de la investigación. Caracas: Ariel.

Schumpeter, J. A. (1944). La teoría del desenvolvimiento económico: Una investigación sobre ganancias, capital, crédito, interés y ciclo económico. México, DF: Fondo de Cultura Económica.

Sullivan, P., \& Kang, J. (1999). Quick response adoption in the apparel manufacturing industry: Competitive advantage of innovation. Journal of Small Business Management, 37, 1-13.

Vila, M., Ferro, C., \& Guisado, M. (2010). Innovación, financiación pública y tamaño empresarial. Cuadernos de Gestión, 10(1), 75-87. 
$\oplus$ 
FORUM EMPRESARIAL

Vol. 19. Núm. 2 • invierno 2014

\title{
Las técnicas financieras para la determinación de valor razonable, su aplicación en los estados financieros y las posibles repercusiones para las empresas
}

\author{
Blanca Iris Vega Castro / blanca.vega@upr.edu \\ Universidad de Puerto Rico, Recinto de Ponce \\ Pedro González Cerrud / drgonzalezcerrud@yahoo.com \\ Universidad de Puerto Rico, Recinto de Río Piedras
}

Recibido: 21 de febrero de 2013

Aceptado: 12 de junio de 2014

$\oplus$

RESUMEN

Este artículo examina la reciente normativa contable internacional, emitida por IASB y FASB, que hace referencia a la medición y divulgación del valor razonable. Se identifican las técnicas de medición aplicadas y los enfoques adoptados por empresas en diferentes países del mundo. Se analiza la aplicación de los criterios y enfoques de medición recientemente sugeridos para la determinación del valor razonable en los estados financieros y las posibles repercusiones que esto puede tener para las empresas.

Palabras clave: normas internacionales de contabilidad, valor razonable, estados financieros

\section{ABSTRACT}

This paper examines recent international accounting standards, issued by the IASB and FASB, which refer to the measurement and disclosure of fair value. We identify measurement techniques and approaches applied by companies in different countries. We also analyze the application of measurement criteria and approaches recently suggested for determining fair value in financial statements and the possible implications for businesses.

Keywords: international accounting standards, fair value, financial statements 